\title{
CORRIGENDUM
}

\section{Childhood abuse and neglect and insecure attachment states of mind in adulthood: Prospective, longitudinal evidence from a high-risk sample_CORRIGENDUM}

\author{
K. LEE RABY, ${ }^{a}$ MADELYN H. LABELLA, ${ }^{b}$ JODI MARTIN,${ }^{c}$ ELIZABETH A. CARLSON, ${ }^{b}$ AND \\ GLENN I. ROISMAN ${ }^{b}$ \\ ${ }^{a}$ University of Utah; ${ }^{b}$ University of Minnesota; and ${ }^{c}$ York University
}

doi:10.1017/S0954579417000037, published by Cambridge University Press, 12 April 2017

We recently identified five cases with minor errors regarding the chronicity of childhood abuse/neglect experiences (no other abuse/neglect parameters were affected by these errors) in our original article (Raby, Labella, Martin, Carlson, \& Roisman, 2017). The correlation between the original and the corrected measure of abuse/neglect chronicity was $r=$ .99 , and regression analyses involving the corrected measure indicated no changes regarding the statistical significance of the associations between abuse/neglect chronicity and the Adult Attachment Interview or Current Relationship Interview dimensions. Updated versions are presented of the three affected tables containing the results related to abuse/neglect chronicity.

In addition, the descriptive information for the abuse and neglect codes originally included on page 351 should be amended as follows:

\section{Reference}

Raby, K. L., Labella, M. H., Martin, J., Carlson, E. A., \& Roisman, G. I. (2017). Childhood abuse and neglect and insecure attachment states of mind in adulthood: Prospective, longitudinal evidence from a high-risk
For the entire Minnesota Longitudinal Study of Risk and Adaptation sample, 47 individuals were classified as being abused and/or neglected in infancy and 66 were classified in early childhood. For the subsample of 164 participants included in the analyses we reported originally (Raby et al., 2017), of those who experienced abuse and/or neglect, $36 \%$ experienced abuse and/or neglect in infancy, 65\% during early childhood, $72 \%$ during middle childhood, and $23 \%$ during adolescence (not mutually exclusive). Regarding chronicity, $34 \%$ of this group experienced abuse and/or neglect during one developmental period, $33 \%$ during two periods, $23 \%$ during three periods, $3 \%$ during all four developmental periods, and $8 \%$ of participants had insufficient data to determine the number of developmental periods (numbers sum to more than $100 \%$ because of rounding).

We regret this error and any problems it may have caused.

sample. Development and Psychopathology, 29, 347-363. doi:10.1017/ S0954579417000037 
Table 3. Correlations among abuse and neglect variables

\begin{tabular}{|c|c|c|c|c|c|c|c|c|}
\hline Variable & 1 & 2 & 3 & 4 & 5 & 6 & 7 & 8 \\
\hline 1. Abuse/neglect status & - & & & & & & & \\
\hline 2. Abuse/neglect chronicity & $.85^{* * *}$ & - & & & & & & \\
\hline 3. No. of abuse/neglect subtypes & $.86^{* * *}$ & $.91 * * *$ & - & & & & & \\
\hline 4. Neglect & $.67 * * *$ & $.75 * * *$ & $.77 * * *$ & - & & & & \\
\hline 5. Sexual abuse & $.51 * * *$ & $.46^{* * *}$ & $.58 * * *$ & .14 & - & & & \\
\hline 6. Physical abuse & $.64 * * *$ & $.67 * * *$ & $.77 * * *$ & $.44 * * *$ & $.16^{*}$ & - & & \\
\hline 7. Paternal abuse perpetrator & $.50 * * *$ & $.52 * * *$ & $.63 * * *$ & $.35 * * *$ & $.37 * * *$ & $.61 * * *$ & - & \\
\hline 8. Maternal abuse perpetrator & $.50 * * *$ & $.57 * * *$ & $.61 * * *$ & $.37 * * *$ & .14 & $.75 * * *$ & $.20 * *$ & - \\
\hline 9. Other abuse perpetrator & $.39 * * *$ & $.33^{* * *}$ & $.39 * * *$ & .09 & $.70 * * *$ & .08 & -.01 & .15 \\
\hline
\end{tabular}

Note: $N=164$.

$* p<.05 . * * p<.01 . * * * p<.001$.

were not included in the composites because the loadings were relatively low. Target participants' CRI dismissing and preoccupied states of mind were positively, but not significantly, correlated $(r=.17, p=.08)$.

\section{Predictive significance of childhood abuse and neglect for adults' attachment states of mind}

The predictive significance of children's experiences of abuse and neglect was evaluated using a set of regression analyses predicting each of the AAI and CRI state of mind dimensions. Separate analyses were conducted for five, nonindependent abuse and neglect parameters: overall classification of abuse/neglect status (binary code indicating whether individuals experienced any type of abuse or neglect during any developmental period), abuse/neglect chronicity (number of developmental periods in which individuals experienced any type of abuse and/or neglect; theoretical range $=0-4$ ), number of abuse and/or neglect subtypes ever experienced (theoretical range $=0-3$ ), specific subtypes (binary codes indicating whether individuals ever experienced neglect, sexual abuse, and/or physical abuse), and abuse perpetrator (binary codes for fathers, mothers, and/or others). These parameters are often included in investigations of the consequences of abuse and neglect for children's development (for a review, see Cicchetti, 2016), including research on AAI outcomes (Roisman et al., 2017 [this issue]).

The various abuse and neglect parameters empirically overlap to a considerable degree (see Table 3 for correlations within the AAI subsample). For each outcome, we began with an omnibus assessment of whether attachment states of mind were predicted by childhood abuse/neglect status, followed by abuse/neglect chronicity and the number of abuse/neglect subtypes. In order to examine what aspects of abuse and/or neglect are most strongly implicated in the development of adult attachment states of mind, we then attempted to decompose these more global associations by focusing on parameters that reflect specific subtypes of childhood abuse and/or neglect experiences as well as specific abuse perpetrators. For each analysis, the focal abuse and neglect variables were entered in the initial step of the regression model. Participant gender, participant ethnicity, maternal education, and family socioeconomic status were also included in the second step. Finally, the nonfocal state of mind dimension was included in a third step of the regression model to test whether the predictive significance of the various abuse and neglect experiences were unique to the attachment state of mind dimension of interest.

Descriptive statistics and zero-order correlations are presented in Table 4. AAI dismissing states of mind were uniquely associated with CRI dismissing states of mind, and AAI preoccupied states of mind were uniquely associated with CRI preoccupied states of mind. Consistent with findings from several other large samples (Haydon et al., 2014; Roisman et al., 2017 [this issue]), males scored higher on dismissing AAI states of mind than did females, and females scored higher on preoccupied AAI states of mind than did males. In addition, participants who were White/non-Hispanic had lower levels of preoccupied AAI states of mind than participants from other ethnic backgrounds.

AAI attachment states of mind. As reported in Tables 5-7, experiencing abuse and/or neglect, especially during multiple developmental periods or involving multiple subtypes, significantly increased risk for AAI preoccupied, but not dismissing, states of mind. These associations were robust to controls for participant gender, ethnicity, maternal education, childhood socioeconomic conditions, and AAI dismissing states of mind. Analyses related to the specific abuse and neglect subtypes indicated that physical abuse was associated with risk for AAI preoccupied states of mind even after controlling for gender, ethnicity, maternal education, childhood socioeconomic conditions, and AAI dismissing states of mind (see Table 8). In addition, there was a marginally significant association between experiencing sexual abuse and increased risk for AAI preoccupied states of mind; however, this association was no longer statistically significant after controlling for covariates. Neglect during childhood was not uniquely associated with increased risk for either AAI preoccupied or dismissing states of mind. Abuse perpetration 
Table 6. Predicting AAI dimensions from the chronicity of childhood abuse/neglect experiences

\begin{tabular}{|c|c|c|c|c|c|c|}
\hline & \multicolumn{3}{|c|}{ AAI Dismissing } & \multicolumn{3}{|c|}{ AAI Preoccupied } \\
\hline & $\beta$ & $p$ & $R^{2}$ & $\beta$ & $p$ & $R^{2}$ \\
\hline 1. Abuse/neglect chronicity & 0.05 & .53 & .00 & 0.23 & $<.01$ & .05 \\
\hline 2. Abuse/neglect chronicity & 0.07 & .91 & .04 & 0.24 & $<.01$ & .15 \\
\hline Participant gender & -0.17 & .02 & & 0.23 & $<.01$ & \\
\hline Participant ethnicity & 0.06 & .48 & & -0.22 & $<.01$ & \\
\hline Maternal education & 0.01 & .99 & & -0.06 & .50 & \\
\hline Childhood SES & -0.09 & .36 & & 0.07 & .43 & \\
\hline 3. Abuse/neglect chronicity & 0.10 & .26 & .14 & 0.24 & $<.01$ & .22 \\
\hline Participant gender & -0.10 & .20 & & 0.17 & .02 & \\
\hline Participant ethnicity & -0.02 & .81 & & -0.20 & $<.01$ & \\
\hline Maternal education & -0.02 & .85 & & -0.06 & .48 & \\
\hline Childhood SES & -0.06 & .49 & & 0.05 & .61 & \\
\hline AAI dismissing & - & - & & -0.30 & $<.01$ & \\
\hline AAI preoccupied & -0.34 & $<.01$ & & - & - & \\
\hline
\end{tabular}

Note: $N=164$. AAI, Adult Attachment Interview; SES, socioeconomic status. For participant gender, $1=$ female, $0=$ male. For participant ethnicity, $1=$ White/non-Hispanic, $0=$ other. All models were significant at $p<.01$ except Model 1 and Model 2 for AAI dismissing ( $p=.47$ and .19 , respectively).

Table 7. Predicting AAI Dimensions from the number of childhood abuse/neglect subtypes

\begin{tabular}{|c|c|c|c|c|c|c|}
\hline & \multicolumn{3}{|c|}{ AAI Dismissing } & \multicolumn{3}{|c|}{ AAI Preoccupied } \\
\hline & $\beta$ & $p$ & $R^{2}$ & $\beta$ & $p$ & $R^{2}$ \\
\hline 1. No. of abuse/neglect subtypes & 0.02 & .86 & .00 & 0.23 & $<.01$ & .05 \\
\hline 2. No. of abuse/neglect subtypes & -0.03 & .74 & .04 & 0.24 & $<.01$ & .15 \\
\hline Participant gender & -0.18 & .02 & & 0.23 & $<.01$ & \\
\hline Participant ethnicity & 0.05 & .49 & & -0.22 & $<.01$ & \\
\hline Maternal education & -0.01 & .93 & & -0.07 & .48 & \\
\hline Childhood SES & -0.10 & .33 & & 0.06 & .52 & \\
\hline 3. No. of abuse/neglect subtypes & 0.06 & .52 & .13 & 0.23 & $<.01$ & .21 \\
\hline Participant gender & -0.10 & .19 & & 0.18 & .01 & \\
\hline Participant ethnicity & -0.02 & .82 & & -0.20 & $<.01$ & \\
\hline Maternal education & -0.03 & .76 & & -0.07 & .44 & \\
\hline Childhood SES & -0.08 & .42 & & 0.03 & .74 & \\
\hline AAI dismissing & - & - & & -0.30 & $<.01$ & \\
\hline AAI preoccupied & -0.33 & $<.01$ & & - & - & \\
\hline
\end{tabular}

Note: $N=164$. AAI, Adult Attachment Interview; SES, socioeconomic status. For participant gender, $1=$ female, $0=$ male. For participant ethnicity, $1=$ White/non-Hispanic, $0=$ other. All models were significant at $p<.01$ except Model 1 and Model 2 for AAI dismissing $(p=.86$ and .19 , respectively).

2014). Specifically, the state of mind scales loaded on two weakly correlated dimensions reflecting dismissing and preoccupied states of mind during the AAI. Moreover, the ratings of unresolved discourse loaded on the same factor as indicators of preoccupied states of mind, providing evidence that preoccupied and unresolved discourse during the AAI reflect a unitary psychological construct even within higher risk samples where these forms of insecurity are more prevalent.

Exploratory factor analyses of the CRI attachment state of mind ratings provided novel evidence regarding the factor structure of individual differences in adults' romantic attachment representations. Paralleling the AAI results, ratings that are traditionally used to differentiate between individuals with secure and dismissing romantic attachment states of mind loaded on one factor, whereas the ratings of adults' preoccupied and unresolved romantic attachment states of mind loaded on a second factor. Taken together, these findings indicate that variation in adults' discourse when discussing attachment-relevant experiences within their childhood caregiving relationships and their current romantic relationships are most parsimoniously captured by two modestly correlated dimensions reflecting dismissing and preoccupied states of mind. In combination with factor analytic findings related to infants' attachment behaviors (Fraley \& Spieker, 2003) and adults' self-reported thoughts, feelings, and behaviors within close relationships (Fraley \& Waller, 1998), these re- 
Table 11. Predicting CRI dimensions from the chronicity of childhood abuse/neglect experiences

\begin{tabular}{|c|c|c|c|c|c|c|}
\hline & \multicolumn{3}{|c|}{ CRI Dismissing } & \multicolumn{3}{|c|}{ CRI Preoccupied } \\
\hline & $\beta$ & $p$ & $R^{2}$ & $\beta$ & $p$ & $R^{2}$ \\
\hline 1. Abuse/neglect chronicity & 0.24 & $<.01$ & .06 & 0.09 & .38 & .01 \\
\hline 2. Abuse/neglect chronicity & 0.22 & .03 & .10 & 0.12 & .26 & .05 \\
\hline Participant gender & -0.15 & .08 & & 0.18 & .05 & \\
\hline Participant ethnicity & -0.07 & .45 & & -0.05 & .59 & \\
\hline Maternal education & -0.14 & .23 & & 0.04 & .73 & \\
\hline Childhood SES & 0.02 & .83 & & 0.03 & .82 & \\
\hline 3. Abuse/neglect chronicity & 0.20 & .04 & .13 & 0.08 & .45 & .08 \\
\hline Participant gender & -0.18 & .04 & & 0.21 & .02 & \\
\hline Participant ethnicity & -0.06 & .51 & & -0.04 & .68 & \\
\hline Maternal education & -0.15 & .20 & & 0.07 & .57 & \\
\hline Childhood SES & 0.02 & .86 & & 0.02 & .86 & \\
\hline CRI dismissing & - & - & & 0.18 & .05 & \\
\hline CRI preoccupied & 0.17 & .05 & & - & - & \\
\hline
\end{tabular}

Note: $N=116$. CRI, Current Relationship Interview; SES, socioeconomic status. For participant gender, $1=$ female, $0=$ male. For participant ethnicity, $1=$ White/non-Hispanic, $0=$ other. Model 1 and 3 for CRI dismissing were significant at $p<.05$ (Model 2, $p=.06$ ). All models for CRI preoccupied were not statistically significant (Model 1, $p=.24$; Model 2, $p=.19$; Model 3, $p=.10$ ).

Table 12. Predicting CRI dimensions from the number of childhood abuse/neglect subtypes

\begin{tabular}{|c|c|c|c|c|c|c|}
\hline & \multicolumn{3}{|c|}{ CRI Dismissing } & \multicolumn{3}{|c|}{ CRI Preoccupied } \\
\hline & $\beta$ & $p$ & $R^{2}$ & $\beta$ & $p$ & $R^{2}$ \\
\hline 1. No. of abuse/neglect subtypes & 0.22 & .02 & .05 & 0.06 & .58 & .01 \\
\hline 2. No. of abuse/neglect subtypes & 0.20 & .04 & .10 & 0.07 & .50 & .04 \\
\hline Participant gender & -0.15 & .09 & & 0.18 & .05 & \\
\hline Participant ethnicity & -0.07 & .44 & & -0.05 & .57 & \\
\hline Maternal education & -0.15 & .18 & & 0.02 & .84 & \\
\hline Childhood SES & 0.01 & .92 & & 0.02 & .89 & \\
\hline 3. No. of abuse/neglect subtypes & 0.19 & .05 & .13 & 0.03 & .76 & .08 \\
\hline Participant gender & -0.19 & .04 & & 0.21 & .02 & \\
\hline Participant ethnicity & -0.06 & .50 & & -0.04 & .66 & \\
\hline Maternal education & -0.16 & .17 & & 0.05 & .65 & \\
\hline Childhood SES & 0.01 & .95 & & 0.01 & .91 & \\
\hline CRI dismissing & - & - & & 0.19 & .04 & \\
\hline CRI preoccupied & 0.18 & .04 & & - & - & \\
\hline
\end{tabular}

Note: $N=116$. CRI, Current Relationship Interview; SES, socioeconomic status. For participant gender, $1=$ female, $0=$ male. For participant ethnicity, $1=$ White/non-Hispanic, $0=$ other. Model 1 and 3 for CRI dismissing were significant at $p<.05$ (Model 2,p=.08). All models for CRI preoccupied were not statistically significant (Model 1, $p=.58$; Model 2, $p=.27$; Model 3, $p=.12$ ).

extends the findings from Roisman et al. (2017 [this issue]) by suggesting that the predictive significance of childhood experiences of abuse and/or neglect for preoccupied states of mind during the AAI may persist into adulthood.

In contrast to the findings from Roisman et al. (2017 [this issue]), associations between abuse and/or neglect experiences and dismissing AAI states of mind in the MLSRA generally were trivial in overall magnitude and consistently were not statistically significant. One possible explanation is that differences in research design or characteristics of the participants involved in the MLSRA and in the Roisman et al. (2017 [this issue]) study may have contributed to the differences in findings from the two studies. Alternatively, the con- sequences of abuse and neglect for dismissing AAI states of mind may be limited to adolescence and may not persist into adulthood. It is necessary to repeatedly collect AAI data across the transition from adolescence and adulthood in a sample with prospective information regarding childhood experiences of abuse and neglect in order to rigorously evaluate whether the predictive significance of childhood abuse and neglect for individuals' dismissing AAI states of mind in particular might fade over this transitional developmental period.

We also sought to extend the research in this area by investigating the predictive significance of specific forms of childhood abuse and neglect for adults' attachment-related representations of their romantic partners. In contrast to the 